\title{
Polyethylene and the Nucleating Agent: Dibenzylidene Sorbitol, a Neutron Scattering Study
}

\author{
Geoffrey R Mitchell ${ }^{1, a^{*}}$, Supatra Pratumshat ${ }^{2, b}$ and Robert Olley ${ }^{3, c}$ \\ ${ }^{1}$ Centre for Rapid and Sustainable Product Development Institute Polytechnic of Leiria Marinha \\ Grande, Portugal \\ ${ }^{2}$ Department of Chemistry, Faculty of Science, Naresuan University Phitsanulok 65000 Thailand \\ ${ }^{3} E M L A B$ University of Reading, Whiteknights, Reading UK \\ ageoffrey.mitchell@ipleiria.pt, bsupatraw@nu.ac.th, 'ogwefan-rho@hotmail.com
}

Keywords: crystallization; morphology; nanoparticles; shear; orientation; small-angle neutron scattering

\begin{abstract}
Dibenzylidene Sorbitol is a very effective clarification additive for isotactic polypropylene. It is not effective with polyethylene although recent studies have shown that it can act as an effective nucleating and directing agent. We employ small-angle neutron scattering measurements to more fully understand the behavior of dibenzylidene sorbitol in a polyethylene matrix. The small-aangle neutron scattering clearly shows that the disappearance of the DBS fibrils is concentration dependent and for $2 \%$ DBS the midpoint of the dissolution is at $145^{\circ} \mathrm{C}$ and for $1 \%$ is $\sim 20^{\circ} \mathrm{C}$ lower
\end{abstract}

\section{Introduction}

Di-benzylidene sorbitol (DBS) [Scheme I] is a widely used low molar mass gelator [1-4]. In a variety of solvents $<1 \mathrm{w} / \mathrm{w} \%$ it self-assembles into extended crystalline nano-fibrils [5]. Similar behavior is observed using some polymers such as polypropylene [6] and poly(e-caprolactone) [7]. When DBS is dispersed in a polymer matrix, the application of modest shear flow leads to a macroscopic alignment of the fibrils $[5,6,7]$. Where the matrix is a crystallisable polymer, subsequent crystallisation of the matrix is templated by the DBS nanofibrils $[5,6]$. In this work we focus on of the nanofibrils. We centre our attention on the behaviour of the DBS in a polyethylene matrix.

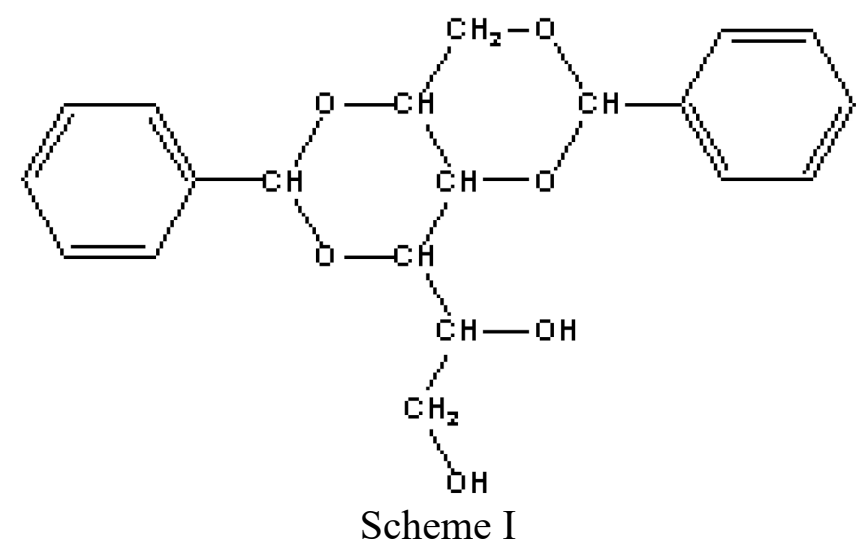

DBS is employed as a clarifying agent with isotactic polypropylene where it yields films with a high level of optical transparency for packaging [8]. It is widely known that DBS with polyethylene is not as effective as a clarifying agent. There is also interest in the use of DBS with polyethylenes used for insulators for power transmission. In the AC cables, the flow of charges inverts its direction too quickly to allow any substantial build up of space charges at the inhomogeneities in the polymeric insulating material. As a consequencethe space charge field can be neglected In recent years high voltage cables have bcome of interest, in part due to the limitations of AC cables to overhead powerlines. In DC cablees the build up of space charges can be disregarded. For semi- 
crystalline polymers, the morphology plays a large part in determing the breaakdown voltage. Li et al [9] have explored the charge distribution in polyethylene as part of a programme focused on the development of insulating materials for power cables. This study concluded that the addition of relatively small quantities of a derivative of DBS lead to reduced space charges and a reduction in the size and number of spherulites. Manaure and Mueller [10] in a study of mixtures of polypropylene and ethylene/ $\alpha$-olefin copolymers concluded that the addition of $1 \% \mathrm{PP}$ and $0.1 \%$ DBS induced a very strong nucleation effect comparable to the effect observed with self-nucleation. Vaughan and Hosier [11] in a detailed study of the effect of DBS on the crystallistaion behaviour of polyethylene found greatly enhanced nucleation with nucleation densities increased by between 2 \& 3 orders of magnitude. However for polyethylene crystallisation at temperatures above $120^{\circ} \mathrm{C}$ the nucleating efficiency of DBS reduces dramatically and the lamellar morphology is replaced by axialites or the early stage of a spherulite structure. They attribute these changes to the relative size of the DBS fibrils. Gherbaz et al studied the effects of composition in the polyethylene and dibenzylidene sorbitol system on the space charge distribution [12] andfound that the addition of $0.3 \%$ DBS produces shallow charge traps but the addition of between 1 and 3\% DBS yielded deep traps. They attributed these to a change in the nature of the DBS fibrils with composition. In the current work we explore the questions raised in these earlier papers on the nature of the interactions between polyethylene and DBS.

Small-angle neutron scattering is a powerful technique in polymer science [13]. It is particular appropriate to the study of large scale structure $(1 \mathrm{~nm}-100 \mathrm{~nm})$ such as the nanoscale DBS fibrils It has the advantage that the scattering contrast can be varied using isotopic substitution, for example, the scattering length of hydrogen is $-3.74 \mathrm{fm}$ while deuterium is $6.67 \mathrm{fm}$. For small-angle x-ray scattering the contrast is fixed by the atomic number and the major contrast arises due to changes in density. This means that it is easy to detect the melting or dissolution of the DBS nano crystalline fibrils [7] but is less straightforward to see whether the DBS and PE form a single solution or a phase separated mixture. We utilize a hydrogenated DBS and a per-deuterated PE to circumvent such problems.

\section{Experimental.}

The 1,3:2,4-dibenzylidene sorbitol (Scheme I) was obtained as Irgaclear D from Ciba Geigy and used without modification. We utilised a perdeuterated linear polyethylene purchased from CK Isotopes Ltd as the matrix with a Mw of 247,053 and a Mn of 23,566. The deuteration is required to provide a contrast for the small-angle neutron scattering measurements. $1 \%$ and $2 \% \mathrm{w} / \mathrm{w} \mathrm{Di}$ benzylidene sorbitol (DBS) was dispersed in the perdeuterated polyethylene (DPE) by solution mixing using xylene as the co-solvent. The solvent was removed by leaving to dry in the fume hood draught.

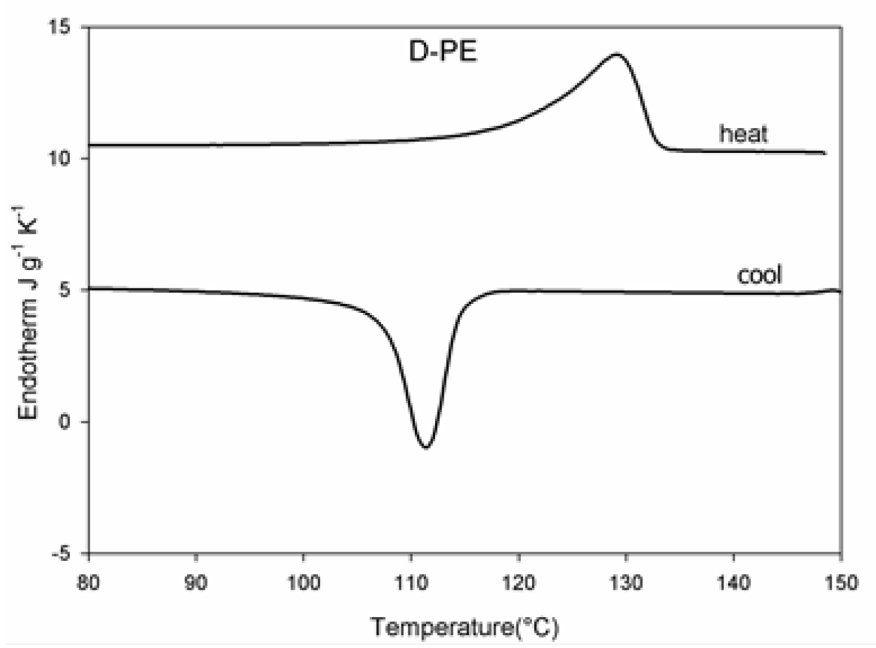

Figure 1 Differential Scanning Calorimetry scans of the perdeuterated polyethylene used in this work. 
Samples of the dried mixtures were formed in to discs using a melt moulding technique. For the $\mathrm{x}$ ray scattering measurements these discs were loaded in to a purpose designed parallel plate shear flow system [14] which facilitated in-situ x-ray and neutron scattering measurements. This is shown schematically in Figure 2. This apparatus allowed the material to be subjected to a programmable temperature profile. DSC scans (Figure 1) exhibit the temperature at the peak of the melting endotherm at $128.5^{\circ} \mathrm{C}$ and the peak of the crystallization endotherm was $110.9^{\circ} \mathrm{C}$.

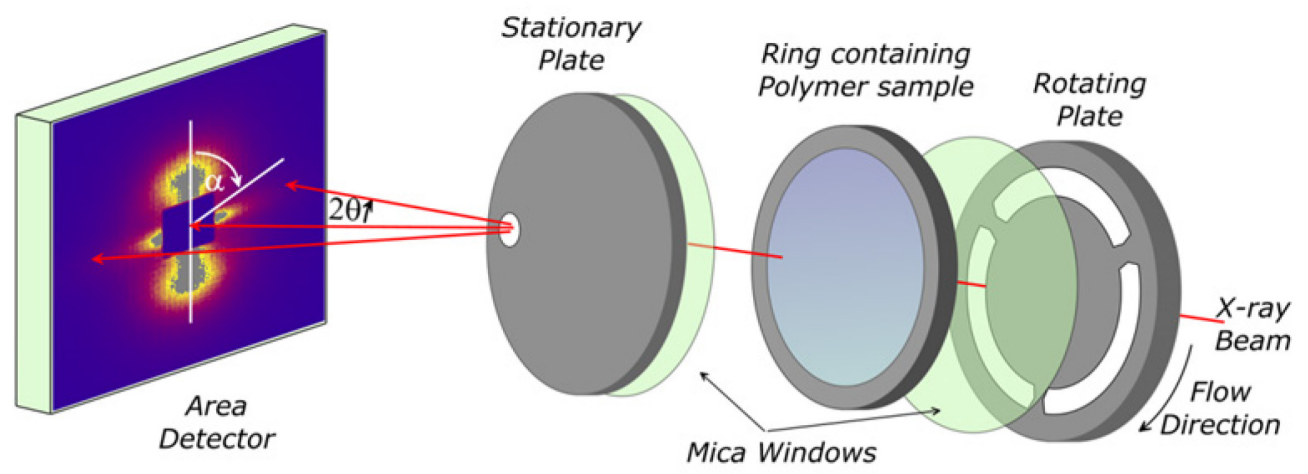

Figure 2 Schematic of the shear cell system [14].

Small-angle neutron scattering (SANS) measurements were performed at the UK ISIS Pulsed neutron facility using the LOQ spectrometer with the sample environment shown in Figure 2. SANS was performed using LOQ at ISIS. A wavelength range of $2.2 \AA-10 \AA$ was employed giving a scattering vector range $|\mathrm{Q}|(\AA-1)$ range of $0.006 \AA-1-0.27 \AA-1$ where

$|\underline{Q}|=4 \pi \sin \theta / \lambda$

and $2 \theta$ is the scattering angle. The SANS Data was processed, including radially averaging, using the COLETTE Software. The scattering amplitude I(Q), is related to the variation in scattering length density by the Equation [1]

$$
I(Q)=\frac{1}{V} \int_{V} \rho(r)\left(e^{i Q r}\right) d r
$$

where $\mathrm{V}$ is the volume, $\mathrm{Q}$ is the modulus of the scattering vector given by $|\mathrm{Q}| 04 \pi \sin (\theta) / \lambda$, $\lambda$ is the incident wavelength and $2 \theta$ the scattering angle and $\rho(r)$ is the spatial variation in the scattering length density in the sample. $\mathrm{n}$ a 2 phase system in which the phases with different scattering length densities $\rho_{1}$ and $\rho_{2}$ rearranged in separate domains with volumes V1 and V2

$$
I(Q)=\frac{1}{V}\left(\rho_{1}-\rho_{2}\right)^{2}\left|\int e^{i Q r} d r_{1}\right|^{2}
$$

Where the first term on the right hand side relates to the differences in neutron scattering length and in small-angle neutron scattering experiments is referred to as the contrast, while the second term describes the spatial arrangement of the mixture. The second term can be written in terms of a single particle shape function $\mathrm{P}(\mathrm{Q})$ often referred to as the form factor, for example a sphere or cylinder. For a concentrated mixture there will be interparticle interactions and these are represented by a function $\mathrm{S}(\mathrm{Q})$. For a dilute system as considered in this work, the interparticle function will be constant and equal to 1 


$$
I(Q)=\frac{N}{V}\left(\rho_{1}-\rho_{2}\right)^{2} V_{P}^{2} P(Q) S(Q)
$$

The form factors for simple shapes are well established [16], for example for a sphere of radius $r$

$$
P(\mid Q) \mid)=\left[\frac{3(\sin (|Q||r|)-|Q||r| \cos (|Q||r|))}{(|Q||r|)^{3}}\right]^{2}
$$

And that for cylinders of radius $r$ and length $L=2 h$

$$
P(|Q|)=\int_{0}^{\pi / 2} f^{2}(|Q|, \alpha) \sin \alpha d \alpha
$$

Where

$$
f(|Q|, \alpha)=J_{0}(|Q| H \cos \alpha) \frac{J_{1}(|Q||r| \sin \alpha)}{|Q||r| \sin \alpha}
$$

Where $\mathrm{J}_{0}$ and $\mathrm{J}_{1}$ are Bessel Functions of the first kind. It is also common to use a log-log plot of the scattered intensity versus the modulus of the scattering vector $Q$ which may enable a power law relationship to be identified and the exponent is related to the fractal number [15].

\section{Results.}

To further explore the dissolution temperature of the DBS in polyethylene we performed smallangle neutron scattering measurements on mixtures of $1 \%$ and $2 \%$ DBS and deuterated polyethylene as a function of temperature. Due to limited beam time we were only able to obtain data at the selected temperatures as shown in Figure 8.
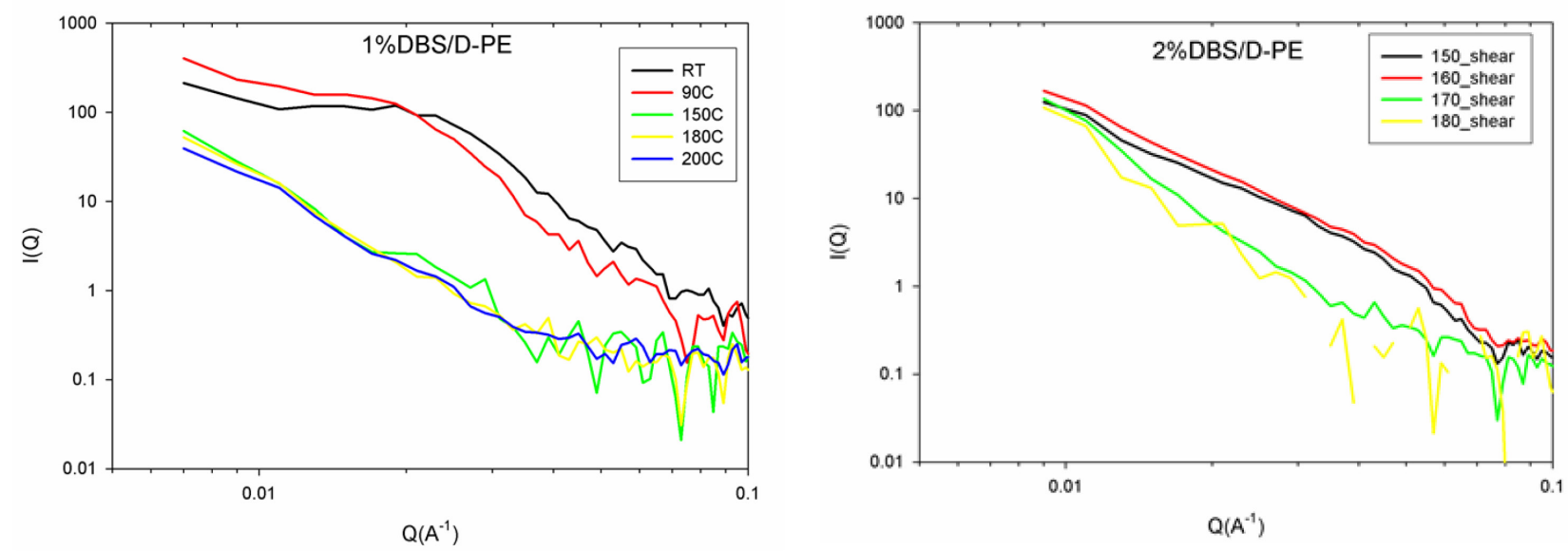

Figure 3 The SANS curves recorded for (a) 1\%DBS /DPE and (b) 2\% DBS/DPE at the temperatures recorded in the legend.

In the case of the curves for $1 \% \mathrm{DBS} / \mathrm{DPE}$ there is marked reduction in the scattered intensity between $90 \mathrm{C}$ and $150^{\circ} \mathrm{C}$. Similar behavior is observed for the $2 \% \mathrm{DBS} / \mathrm{DPE}$ (Figure $3 \mathrm{~b}$ ). 
To quantify these changes we calculated the so-called invariant (Equation 6) which is directly related to the average of the square of the scattering length density differences [7]; if the density of the fibrils are constant this is proportional to the volume fraction of the fibrils.

$$
\Omega=\int_{0}^{\pi / 2} \int_{Q=0}^{Q_{\max }}|Q|^{2} I(|Q|, \alpha) \sin \alpha d Q d \alpha
$$

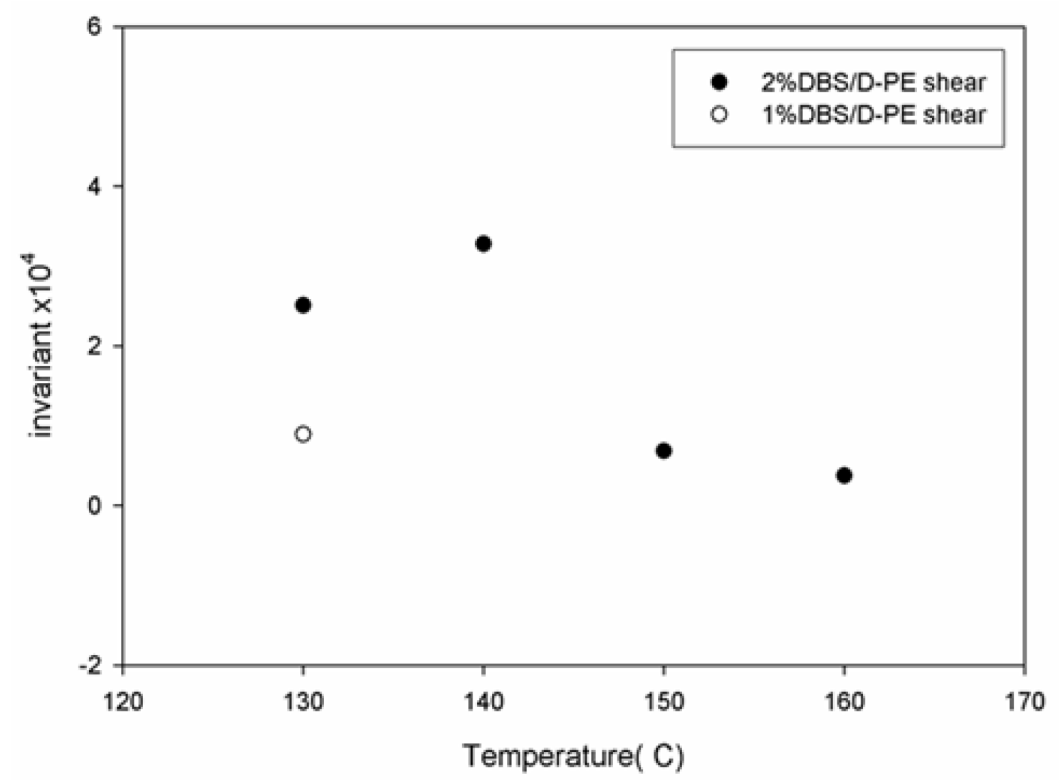

Figure 4 The invariant calculated for each scattering curve plotted as function of the experimental temperature. Filled circles $2 \%$ DBS/DPE, open circle 1\%DBS/DPE.

We can see that by $130^{\circ} \mathrm{C}$ the invaraiant for the $1 \%$ DBS/DPE has fallen to a value close to zero. For the $2 \% \mathrm{DBS} / \mathrm{DPE}$ the equivalent point is $150^{\circ} \mathrm{C}$. The midpoint of the transition observed in the invariant for $2 \% \mathrm{DBS} / \mathrm{DPE}$ is $\sim 145^{\circ} \mathrm{C}$ and we estimate that the equivalent point for $1 \% \mathrm{DBS} / \mathrm{DPE}$. Is $\sim 125^{\circ} \mathrm{C}$. As these values are different for the 2 concentrations we can deduce that the process is one of dissolution of the fibrils in the DPE rather than a melting process. The rapidly reducing nucleation efficiency of DBS above $120^{\circ} \mathrm{C}$ reported by Vaughan and Hosier [11] we attribute to the reduction in the volume fraction of DBS fibrils present in the sample and available to act as nucleants.

From the slope of the curves on a log-log plot as shown in Figure 3 we can derive a fractal number which provides a clue to the morphology of the DBS fibrils. The value obtain is $\sim 3$ indicaating a $3 \mathrm{~d}$ spacing filling structure which suggests much branching. Of course here the fibrils do not have a common alignment induced by shear flow as observed in previous work [16]. Using the low $\mathrm{Q}$ portion of the curves, as described in [16] we calculate the radius of gyration to be $15 \mathrm{~nm}$, a value similar to observed in earlier work [5] .

\section{Summary}

Small-angle neutron scattering measurements of low fractions of DBS in a perdeuterated matrix have confirmed the basic model identified in earlier work using x-ray scattering. At high temperatures the DB/DPE form a single phase state in which the DBS is dissolved in the DPE: At lower temperatures the DBS forms fibrils which in heating dissolve back in to the polymer matrix at a temperature dependent on the composition. For $2 \% \mathrm{DBS} / \mathrm{DPE}$ this temperature is $145^{\circ} \mathrm{C}$ for $1 \%$ DBS/DPE we estimate that temperature to be $\sim 125^{\circ} \mathrm{C}$. 


\section{Acknowledgements}

This research was supported by the European Regional Development Fund (FEDER), through COMPETE2020 under the PT2020 program (POCI-01-0145-FEDER-023423), by the Portuguese Foundation for Science and Technology (UID/Multi/04044/2013), (UC4PE and Centro2020 PAMI - ROTEIRO/0328/2013 (No 022158).. The SANS measurements were made at the STFC ISIS Facility using the LOQ instrument with the help of ISIS Staff. We are grateful to Dr Stephen King for his help. We thank Naresuan university for supporting SP during her Ph.D. and Faculty of Science Naresuan University for funding short visits.

\section{References}

[1] D.K.Smith, Chem.Soc. Rev., 2009 38, 684-694.

[2] N.M.Sangeetha, U.Maitra, Chem. Soc. Rev., 2005, 34, 821-836.

[3] R.G.Weiss and P.Terech, Molecular Gels: Materials with Self-Assembled Fibrillar Networks, Chapter 8, 233-244, 2006, Springer

[4] P.Terech, R.G.Weiss, Chem. Rev., 1997, 97, 3133-3159

[5] Aurora Nogales, Geoffrey R. Mitchell, and Alun S. Vaughan Macromolecules, 2003, 36, $4898-4906$

[6] A. Nogales, R.H. Olley,and G.R. Mitchell, Macromol. Rapid Comm., 2003, 24, 496-502.

[7] G.R. Mitchell, Supatra Wangsoub, Aurora Nogales, F.J Davis and, R.H. Olley 'Controlling Morphology Using Low Molar Mass Nucleators' in "Controlling the Morphology of Polymers: Multiple Scales of Structure and Processing." Springer 2016 eds G.R.Mitchell and A.Tojeira ISBN 978-3-319-39320-9

[8] Plastics Additives Handbook ed. H.Zweifel Hanser 2001 Chapter 18

[9] Li X, Cao Y, Du Q, Yin Y, Tu D (2001) J Appl Polym Sci 82:611

[10] Manaure AC, Müller AJ (2000) Macromol Chem Phys 201:958

[11] Vaughan, A.S. \& Hosier, I.L. J Mater Sci (2008) 43: 2922. https://doi.org/10.1007/s10853007-1801-9

[12] G. Gherbaz, G. Chen, and A. Vaughan, Conference on Electrical Insulation and Dielectric Phenomena, Quebec, Canada IEEE, 2008, 161-164.

[13] S.M. King, chap 7 Small Angle Neutron Scattering, Modern techniques for polymer characterization, edited by R.A. Pethrick, J.V. Dawkins, 1999, John Wiley \& Sons

[14] A.Nogales, S.A.Thornley and G.R.Mitchell, J Macromol Sci-Physics 2004, B43, 1161-1170

[15] R-J Roe, Methods of X-ray and Neutron Scattering in Polymer Science, 2000 Oxford University Press, N.Y..

[16] J. Siripitayananon, S. Wangsoub, R.H. Olley, and G.R. Mitchell, Macromolecular Rapid Comm., 2004, 25, 1365- 1370. 